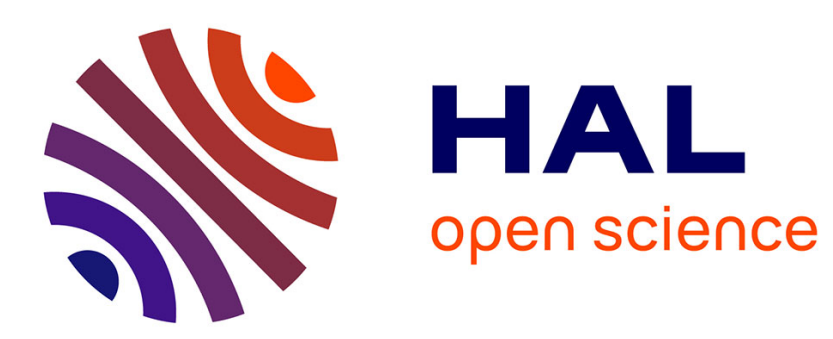

\title{
Micromodel-based simulations for laminated composites
}

\author{
David Violeau, Pierre Ladevèze, Gilles Lubineau
}

\section{To cite this version:}

David Violeau, Pierre Ladevèze, Gilles Lubineau. Micromodel-based simulations for laminated composites. Composites Science and Technology, 2009, 69 (9), pp.1364-1371. 10.1016/j.compscitech.2008.09.041 . hal-01648326

\section{HAL Id: hal-01648326 \\ https://hal.science/hal-01648326}

Submitted on 18 Dec 2019

HAL is a multi-disciplinary open access archive for the deposit and dissemination of scientific research documents, whether they are published or not. The documents may come from teaching and research institutions in France or abroad, or from public or private research centers.
L'archive ouverte pluridisciplinaire HAL, est destinée au dépôt et à la diffusion de documents scientifiques de niveau recherche, publiés ou non, émanant des établissements d'enseignement et de recherche français ou étrangers, des laboratoires publics ou privés. 


\title{
Micromodel-based simulations for laminated composites
}

\author{
David Violeau ${ }^{\mathrm{a}}$, Pierre Ladevèze ${ }^{\mathrm{a}, \mathrm{b}}$, Gilles Lubineau ${ }^{\mathrm{a}, *}$ \\ ${ }^{a}$ LMT-Cachan (E.N.S. Cachan/Paris 6 Univ./C.N.R.S./UniverSud Paris), 61 Avenue du Président Wilson, Cachan Cedex 94235, France \\ ${ }^{\mathrm{b}}$ EADS Foundation Chair, Adv. Computational Structural Mechanics
}

\begin{abstract}
We develop a calculation strategy for the simulation of a complete microscopic model. This strategy enables one to account for damage mechanisms in laminated composites. The model mixes discrete and continuous approaches by introducing potential rupture surfaces and a damageable continuous medium. This approach requires suitable calculation tools unavailable in industrial analysis codes. The strategy presented is multiscale in space and is based on a decomposition of the domain into substructures and interfaces. This strategy enables one to simulate complex problems with multiple cracks. In practice, to use such a model, the strategy must be improved in order to handle very large numbers of substructures and interfaces and to estimate the rupture criteria for the surfaces introduced into the model. We provide simple examples which demonstrate the capabilities of the microscopic model.
\end{abstract}

\section{Introduction}

Today, the design and construction of a structure using composite materials involves numerous and costly tests. A major concern of industry is to replace some of these real tests by numerical simulations. This approach, called virtual testing, requires models and simulations which certification agencies can have confidence in. Laminated composites, the types of materials considered in this work, have been the subject of a very large number of research studies over the past thirty years or so. Today, the degradation mechanisms of these materials are very well-known. Nevertheless, their simulation up to rupture remains difficult and still requires more elaborate models and simulations. Two main families of approaches to the modeling of the degradations in laminated composites are in competition. On the one hand, the micromechanics approach relies on a clearly identified microscopic phenomenology and enables a precise analysis of damage. However, it is limited to simple stratifications and relies on a number of simplifying assumptions which do not lend themselves to structural analysis. On the other hand, the mesomechanics approach models degradations through continuous damage variables representing the stiffness reductions consecutive to damage. Thus, it becomes possible to carry out calculations on arbitrary structures, but the phenomenological interpretation of the results remains difficult. Recently, a complete microscopic model was proposed in [1] and developed in $[2,3]$. This model enables one to describe the different degradation modes through a partly discrete, partly continuous represen-

\footnotetext{
* Corresponding author. Tel.: +331474024 02; fax: +33147402785.

E-mail addresses: ladeveze@lmt.ens-cachan.fr (P. Ladevèze), lubineau@lmt.enscachan.fr (G. Lubineau).
}

tation. This model requires suitable calculation tools in order to simulate simple test samples. The objective of this paper is to present the different numerical aspects involved in the implementation of the microscopic model.

First, we present very quickly the different degradation mechanisms. A complete review of these mechanisms can be found in our previous publications for further details. Some key aspects of the microscopic model are also presented. It is very clear that such a fine modeling needs a very fine discretization of the structure. One has so to deal with large non-linear problems which can not be achieved with the traditional numerical approaches.

In the second part, we give a short description of the multiscale calculation strategy with homogenization in space which is choosen here. This strategy enables one to address complex nonlinear problems. However, several improvements are necessary in order to adapt this strategy to the microscopic model and take into account a very large number of substructures. An additional numerical aspect which is discussed concerns the determination of the rupture criteria introduced to break the surfaces of the model.

Finally, a few examples demonstrate the capabilities of the microscopic model. Although very partial, these first results are promising for the treatment of very strong, localized and coupled discrete degradations within laminated composites.

\section{A brief review of the hybrid micromechanical modeling}

\subsection{Microphenomenology}

Even in the case of simple macroscopic loading and simple geometries, the final state of damage of a composite part appears 


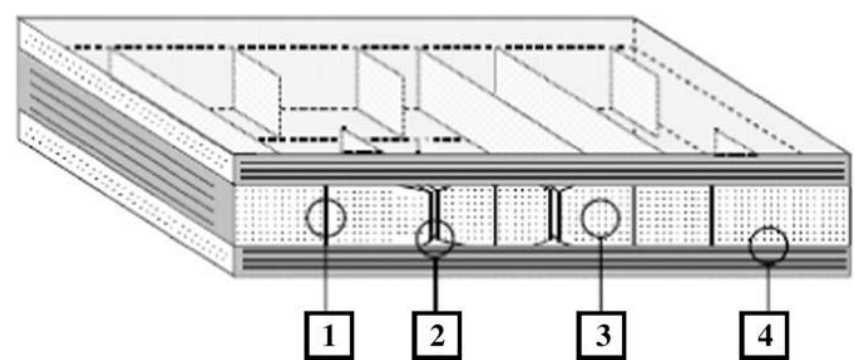

Fig. 1. The mechanisms of degradation on the microscale: 1-transverse microcracking; 2-local delamination; 3-diffuse damage; 4-diffuse delamination.

to be complex. Regardless of the apparent complexity of the degradation patterns, experimental observations show that some major and well-identified mechanisms stand out.

Therefore, our approach consists in assuming that any complex state of degradation is the superposition of simple mechanisms (Fig. 1). First, we will review these mechanisms briefly, following a classification related to the scale of each mechanism - the fine scale (of the order of the diameter of one fiber) and the scale of the ply. We will see later how this classification is used in the model in order to describe each mechanism appropriately.

\subsubsection{Degradation mechanisms on the fine scale}

The following two mechanisms (diffuse intralaminar and interlaminar damage) are usually not introduced in micromechanics, but have been taken into account for many years through continuous damage mechanics, both for the ply [4] and for the interface [5], in the damage mesomodel for laminates introduced in [6]. Models which do not take these mechanisms into account are unreliable under multiaxial loading, especially when high shear loads are applied.

Diffuse intralaminar damage is associated with fiber/matrix debonding within the ply and with small cracks within the matrix. Locally, these degradations are distributed more or less homogeneously throughout the thickness of the unidirectional ply. This mechanism is often omitted in micromechanics and only few directly related observations have been reported $[7,8]$. Nevertheless, a significant decrease in stiffness with no visible transverse cracking can occur due to this mechanism. This is particularly true of shear loading where it is the main mechanism [9]. We also believe that this mechanism is responsible for the high amount of plasticity which affects these materials under shear loading, due to friction between extensively debonded surfaces.

Diffuse interlaminar damage is associated with microvoids within the matrix of the interlaminar interface. It results in seemingly reduced stiffness of the interlaminar interface with no visible delamination.

\subsubsection{Degradation mechanisms on the ply's scale}

Most of the works in micromechanics concentrate on two mechanisms on the scale of the elementary ply.

Transverse microcracking concerns cracks which spread completely across the thickness of the elementary ply. A typical topic of interest in micromechanics is the prediction of the multiplication of these cracks in cross-ply laminates, where they form a quasi-periodic network within the $90^{\circ}$ ply. They occur relatively early in the case of transverse loading, but very late in the case of shear loading. They generally initiate at the edge of the samples, then propagate in a stable or unstable way towards the core [10] depending on the type of ply (the well-known thickness effect [11]) and on the loading (static or cyclic [12]).

As the loading increases, "local delamination" occurs at the interfaces between plies $([13-15], \ldots)$ due to the induced overloading at the tips of the transverse microcracks. In practice, the development of this mechanism is responsible for the saturation of the microcracking state and participates in the complete failure of the structure as a result of the localization of the degradation.

Contrary to the diffuse mechanisms, transverse microcracking and local delamination are characterized by rupture surfaces which are clearly identifiable on the ply's scale and, therefore, have been studied mainly within the framework of finite fracture mechanics. The relative ease with which they can be observed explains the large number of works in micromechanics dealing with their modeling (see $[16,17]$, for reviews).

\subsection{The computational damage micromodel for laminates}

The computational damage micromodel for laminates was introduced in [1]. Its foundations in terms of material behavior are detailed in [2], so we will review only its main concepts. The aim of this approach is to propose a micromechanical model of the mechanisms presented in Section 2.1 which could be used as a reference material under general loading. In that sense, this approach differs drastically from classical micromechanical approaches, which describe only certain specific mechanisms under specific types of loading.

The key point of this approach is the treatment of the mechanisms according to their characteristic scales. On the scale of the elementary ply, diffuse intra- and interlaminar damage can be viewed as locally homogeneous, whereas transverse microcracking and diffuse delamination can be associated with well-defined fracture surfaces. This leads to the following choice: the degradation of the fiber-matrix material is described using the classical continuum mechanics framework, while transverse microcracking and local delamination are described using a discrete model based on a generalization of finite fracture mechanics [18].

\subsubsection{The classical damage mesomodel for diffuse mechanisms}

For diffuse mechanisms, the classical form of the damage mesomodel is used to describe damage associated with fiber/matrix debonding and small cracks within the matrix connecting neighboring fibers (details can be found in [4,5]). For the elementary ply, for instance, the damaged material is still assumed to be transverse-isotropic and its state is described classically by two damage variables, one in the transverse direction and the other in the shear direction, which are assumed to be constant within the thickness of the mesoconstituent.

(Visco)plasticity within the single layer and diffuse damage within the interlaminar interface can be introduced in the same way.

It should be pointed out that contrary to the complete classical damage mesomodel for laminates [4], we introduce no threshold for the damage force. Such a threshold had been initially introduced in order to model the rapid degradation associated with transverse microcracking (local delamination and rupture), but in the current approach presented here this threshold is no longer necessary because these mechanisms are described accurately through finite fracture mechanics, as described in the following section.

\subsubsection{Finite fracture mechanics for transverse microcracking, delamination and fiber breakage}

The surfaces of cracks are described using a discrete model. Thus, the complex cracking pattern within the structure is assumed to result from the complete breakage of elementary surfaces and volumes, entities chosen on the basis of micromechanical considerations [2]. Thus, the mechanisms of degradation are quantified, which guarantees that the approach performs well with respect to localization. 
Two families of surfaces must be considered. The first family is composed of the intralaminar surfaces, which are assumed to be parallel to the fibers and represent transverse cracking. The second family is composed of the interlaminar surfaces, which represent local delamination. The characteristic sizes of the surfaces in each family are defined by micromechanical considerations. For instance, for intralaminar cracks, the minimum cracked surface is a square whose dimension is equal to the thickness of the ply. This choice derives from the fact that for values equal to or greater than the thickness the tunneling energy release rate is nearly constant $[2,19]$.

Whatever the nature of the surface, its failure is based on a discrete energy criterion. Consequently, the basic material quantities involved in the model are the critical energy release rates designated, in each mode, by $\left\{G_{c p}^{I, I I I I I I}\right\}$ for the ply and $\left\{G_{c i}^{I, I I, I I I}\right\}$ for the interface. A surface breaks when its damage force reaches its critical energy release rate. When this happens, friction is introduced.

For the ply as well as for the interface, the damage force $\left\{G_{p}\right\}$ or $\left\{G_{i}\right\}$ is directly related to the classical discrete energy release rate $\left\{\Delta G_{p}\right\}$ or $\left\{\Delta G_{i}\right\}$. In a sense, this is a $2 \mathrm{D}$ generalization of the discrete energy release rate introduced by [18].

For the ply, we distinguish between crack initiation and crack propagation [20] in order to take into account classical experimental observations: thickness effects [11] and local cracks near the edges [10]. Therefore, the damage force is defined as:

- for crack initiation:

$\left\{G_{p}\right\}=\min \left[\left\{\Delta G_{p}\right\} ; \bar{h} \cdot \frac{\left\{\Delta G_{p}\right\}}{h}\right]$

- for crack propagation:

$\left\{G_{p}\right\}=\left\{\Delta G_{p}\right\}$

For fiber breakage, volumes of size $H$ (the thickness of a ply) are considered and an energy criterion is used.

\section{The numerical strategy for the hybrid micromechanical modeling}

The strategy used must allow the introduction of nonlinearities (contact on the surface level, damage of the medium) with relative ease. The approach introduced in [21] and extended in [22,23] relies on a mixed domain decomposition with spatial homogenization. Here, it is extended to three-dimensional media containing multiple cracks.

The domain is divided into substructures and interfaces between these substructures. Each entity has its own variables, equations and constitutive relations. Thus, one can introduce perfect interfaces into the healthy zones and interfaces of the contact type with friction, using a Coulomb law, into the cracked zones.

\subsection{Implementation of the micro model}

The introduction of minimum cracking surfaces could take place in the course of the calculation, which would require one to deal with "dynamic" occurrence and to remesh some zones of the structure each time a crack appears. For the time being, it is easier to define minimum cracking surfaces a priori (see Fig. 2). In the future, one could try to treat this problem without predefining the cracked surfaces.

In order to be able to take into account many possible degradation paths, one must introduce a huge number of minimum cracking surfaces, which leads to prohibitive calculation costs when using the current industrial codes. For example, a $10-\mathrm{cm}$ wide holed structure with $\mathrm{n}$ plies of thickness $\mathrm{H}$ requires the introduction of nearly 1 billion minimum cracking surfaces.

For problems involving several million surfaces, a dedicated method is required. This is also the case for nonlinear problems (for instance, in the presence of a cracking surface, contact with friction can occur). We adopted the multiscale strategy with homogenization described previously. The domain decomposition is chosen in order to be compatible with the minimum cracking surface. Only sequences containing four different orientations (such as $0^{\circ}, 90^{\circ}$ and $\pm \alpha^{\circ}$ ) can be simulated, but this is the case of the great majority of laminated composite structures.

\subsubsection{Improvement of the multiscale strategy}

Due to the sizes of the substructures and interfaces chosen in order to be compatible with the microscopic model, the size of the macro problem, which is related to the number of interfaces, can rapidly become huge. Since the macroscopic problem is linear, the approach we chose consists in seeking an approximate solution. Since the macro problem is formulated through a macro multiplier which tends to zero as the process converges, any approximate solution of this problem enables convergence towards the solution. Only the convergence rate can be seriously affected if the approximation is poor.

The approximation chosen consists in grouping interfaces and substructures into "supermacro cells" denoted $\omega$ (Fig. 3). On each supermacro interface between supermacro cells, one seeks an affine approximation similar to the macro multipliers. One defines an operator which, using the supermacro displacements denoted $\widetilde{W}^{S M}$, enables the determination of the multipliers $\widetilde{W}^{M}$ at the interfaces of a supermacro cell given the supermacro displacements at the interfaces of a supermacro cell.

$$
\widetilde{W}_{E}^{M}=\mathbf{P}_{\partial \Omega_{E}}^{\partial \omega}\left(\widetilde{W}_{\omega}^{S M}\right)
$$
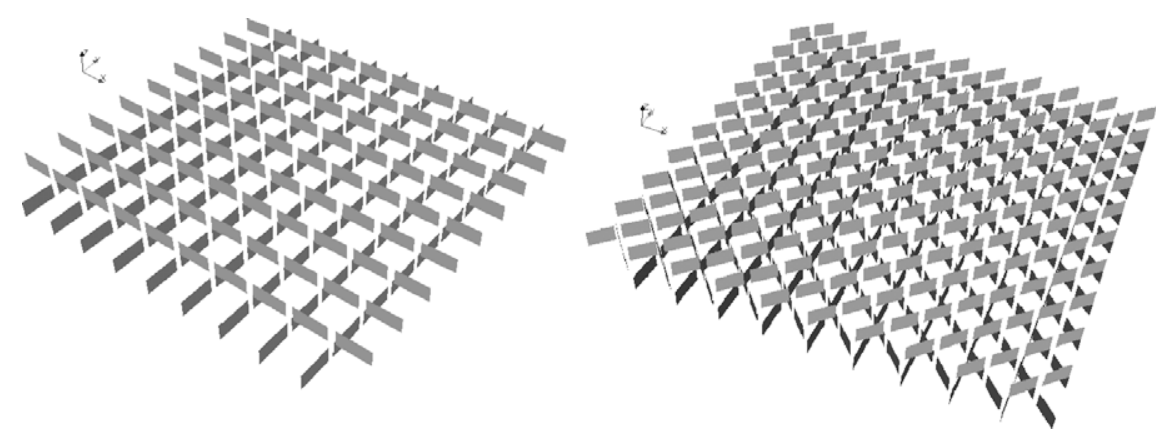

Fig. 2. Predefined potential cracks for $[0,90]_{s}$ and $[45,-45,90]_{s}$ laminates. 


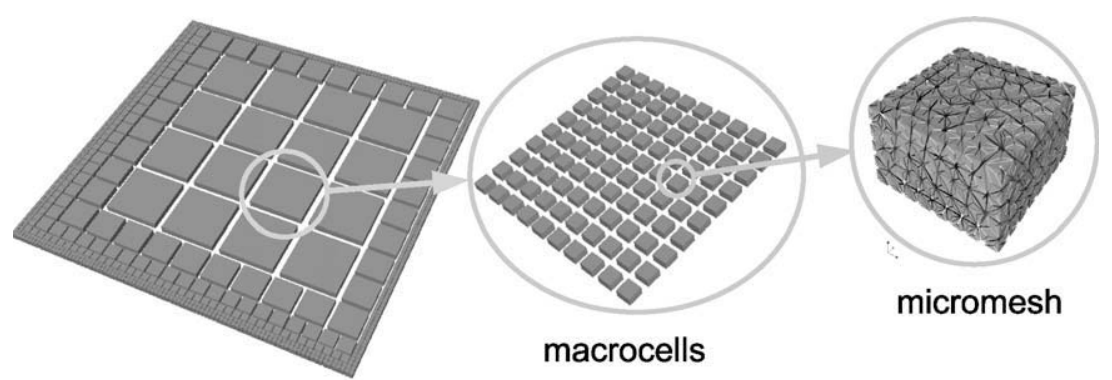

Super-macrocells

Fig. 3. Supermacro cells containing elementary cells.

a

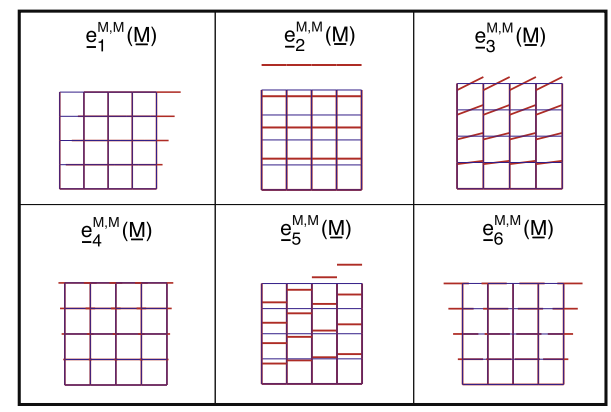

b

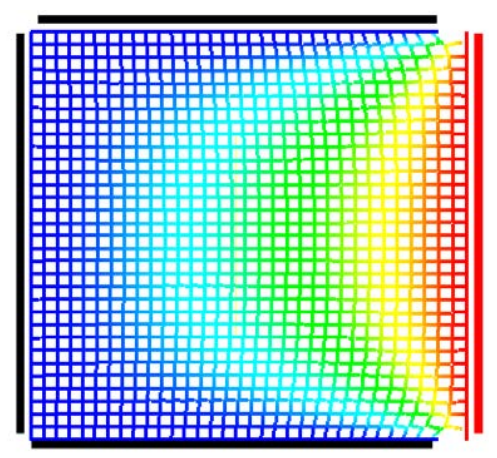

Fig. 4. Supermacro approximations.

Thus, the approximate macro problem in displacements is:

$$
\begin{aligned}
& \text { Find } \underline{\widetilde{W}}^{S M}=\left\{\underline{\widetilde{W}}_{\omega}^{S M}\right\}_{\omega} \in \mathscr{W}_{a d, 0}^{M} \text { which verifies : } \\
& \begin{aligned}
\forall \widetilde{W}_{\omega}^{S M *} \in & \mathscr{W}_{a d, 0}^{M}, \sum_{\omega} \in \mathbf{\Omega} \sum_{E \in \omega} \int_{\partial \Omega_{E}} \mathbf{P}_{\partial \Omega_{E}}^{\partial \omega}\left(\widetilde{W}_{\omega}^{S M *}\right) \\
& \cdot\left(\mathbf{L}_{E}^{F}\left(\mathbf{P}_{\partial \Omega_{E}}^{\partial \omega}\left(\widetilde{W}_{\omega}^{S M}\right)\right)+\underline{F}_{E d}^{M}\right) \mathrm{d} \Gamma \\
& =\sum_{\omega} \in \mathbf{\Omega} \sum_{E \in \omega} \int_{\partial \Omega_{E} \cap \partial \Omega_{F}} \mathbf{P}_{\partial \Omega_{E}}^{\partial \omega}\left(\underline{W}_{\omega}^{S M *}\right) \cdot \underline{F}_{d} \mathrm{~d} \Gamma
\end{aligned}
\end{aligned}
$$

A first method to determine Operator $\mathbf{P}_{\partial \Omega_{\mathrm{E}}}^{\partial \omega}$ consists, starting from a given displacement of a supermacro interface between two supermacro cells, in choosing a particular kinematics of the interfaces. Another technique consists in solving the macro problem in a supermacro cell subjected successively to given $\widehat{W}_{\omega}^{S M}$ (Fig. 4).

A force approach was also developed, but even though it is very efficient in low-gradient zones it is difficult to implement and it leads to only an approximate solution, whereas the displacement approaches converge towards the exact solution.

The following example in 2D shows a comparison of the different approaches applied to a simple bending problem (Fig. 5). We compared the quasi-exact approach (in which the macro problem is first solved in supermacro cells), the Cosserat-type approach (in which a kinematics is used to determine the displacements of the macro interfaces given the supermacro displacements), a force approach using an analytical construction of the forces, and the exact approach. In all the examples, the quasi-exact approach has the best convergence rate, but with a proper discretization of the supermacro cells the convergence rates of the other approaches are comparable and the size of the problem to be solved is smaller by an order of magnitude.

However, the drawback of this technique is that the supermacro partitioning must be fitted to the singularities (i.e. cracks) and ad- justed during the calculation if necessary. Another option would be to solve the macro problem through a domain decomposition method, such as FETI or BDD [24,25].

\subsubsection{Failure criterion}

Besides the number of complete calculations required, an additional difficulty is the determination of the criterion for each potential rupture surface. This criterion is obtained from the recovery rate associated with each mode. Thus, it is necessary to carry out for each potential rupture surface a complete calculation in which the surface considered is either healthy or ruptured. The energy recovery rate is estimated from the potential energy difference between these two configurations.

$\Delta G=-\frac{\Delta \Pi}{\Delta S}$

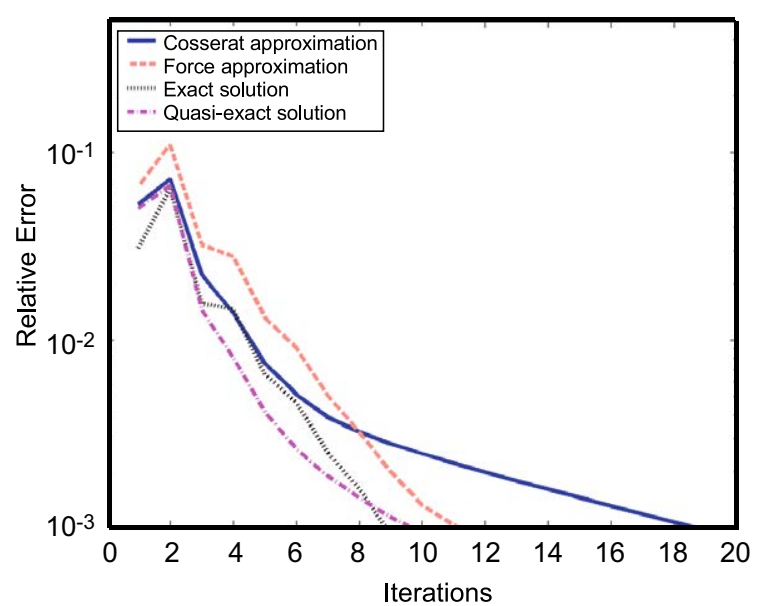

Fig. 5. Comparison of different supermacro approximations and the exact solution. 
where $\Delta \Pi$ is the potential energy difference between two states where one surface is broken, and $\Delta S$ is the area of the broken surface.

Such a calculation requires an entirely new analysis for each potential interface, which is too expensive. Furthermore, it is difficult to extract modes from this global energy release rate.

Then, the idea is to determine an approximation of $\Delta G_{i}$ over each mode. (An a priori verification of the values obtained can be made once the surface is ruptured.) In order to do this, one uses the decomposition of the stresses into an initial part and a residual part proposed by Nairn [26].

Having obtained the solution for a structure in which there are some broken interfaces (State A), one wants to know State B which corresponds to the failure of one selected surface. The calculation in State A gives the stress state $\sigma_{A}$. One can assume that the stress in State B is the sum of two contributions: $\sigma_{B}=\tilde{\sigma}+\sigma_{A}$. The only purpose of this assumption is to have an approximation of the stress state in the next configuration and to calculate the energy release rate. Then, the problem consists in finding the stress correction field $\tilde{\sigma}$ (residual problem). At the selected interface, the traction in State $B$ is zero (i.e. the interface fails). Therefore, the stress at Interface $\tilde{\sigma}$ is $\tilde{\sigma}=-\sigma_{A}$. If one calculates the solution of the residual problem for which $-\sigma_{A} \vec{n} . \vec{x}_{i}$ is applied to the selected interface with zero boundary conditions on the structure, one can obtain the stress correction $\tilde{\sigma}$ on the whole structure for a specific mode (in the direction $\vec{x}_{i}$ ). Then, the energy release rate for one mode can be obtained by calculating the potential energy in State B.

The tractions applied at interface $-\sigma_{A} \vec{n} \cdot \vec{x}_{i}$ have zero resultant and moment. According to Saint-Venant's principle, the effect of this stress is local. The solution of the residual problem can be determined by considering only a small region around the interface. Free tractions are applied along the edge of this local zone (see Fig. 6).

This localized problem is solved using the multiscale strategy (all the local operators have already been determined and the contact conditions can easily be dealt with).
Even though the cost of a local problem is small, a large number of such problems must be solved in order to estimate all the recovery rates. In order to reduce the number of calculations, one determines the recovery rates only in the zone surrounding a newly ruptured surface.

Another improvement consists in storing the results (i.e. the pattern of the ruptured and healthy surfaces) obtained for a given configuration. When the same configuration reappears, one reuses the stored values directly by adjusting them to the mean forces obtained at the surface being considered (see Fig. 7). This "handbooktype" approach is particularly recommended in the case of the propagation of unstable cracks or when the degradation patterns are similar: then, the calculation time for the recovery rates can be reduced by a factor of 100-1000.

\subsubsection{The resolution algorithm}

The resolution algorithm consists in carrying out, for a given loading, a multiscale calculation. Then, the criterion for all poten-

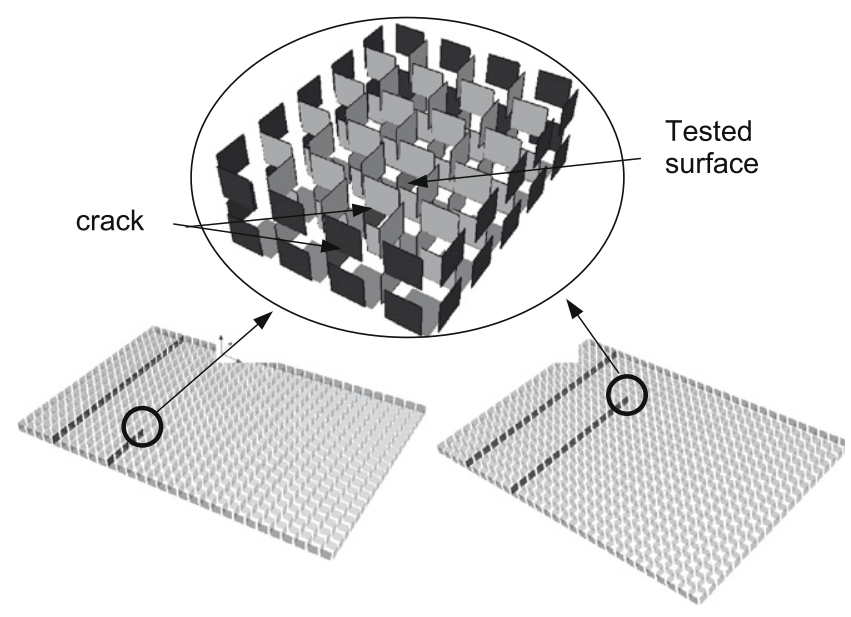

Fig. 7. Reuse of the localized solution.

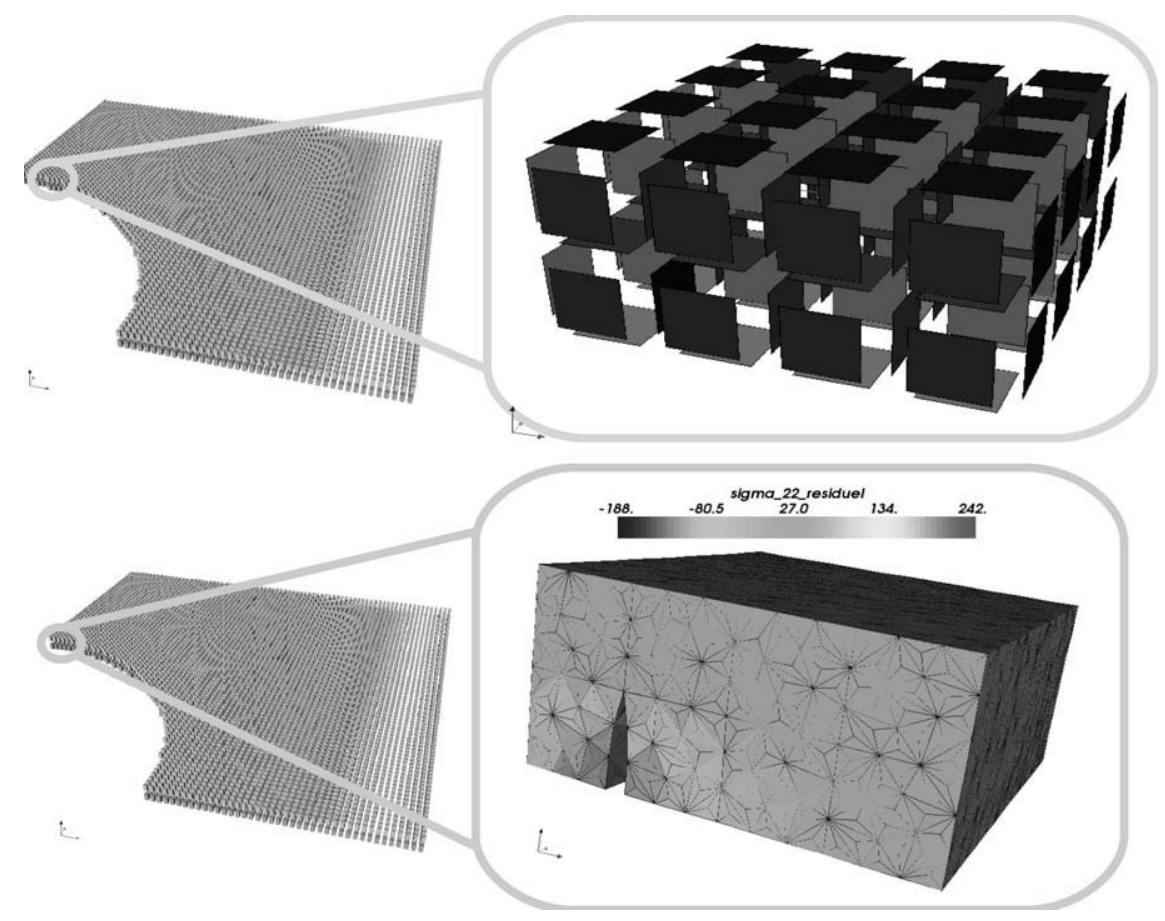

Fig. 6. Localized problem and residual solution. 

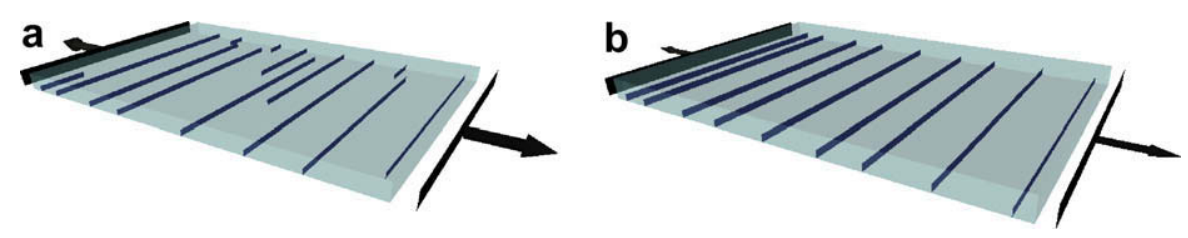

Fig. 8. $[0,90]_{\mathrm{s}}$ laminates (a: thin ply $[0 / 90 / 0]$ (1.15\% strain); b: thick $90^{\circ}$ ply $\left[0_{3} / 90_{3} / 0_{3}\right](0,7 \%$ strain).
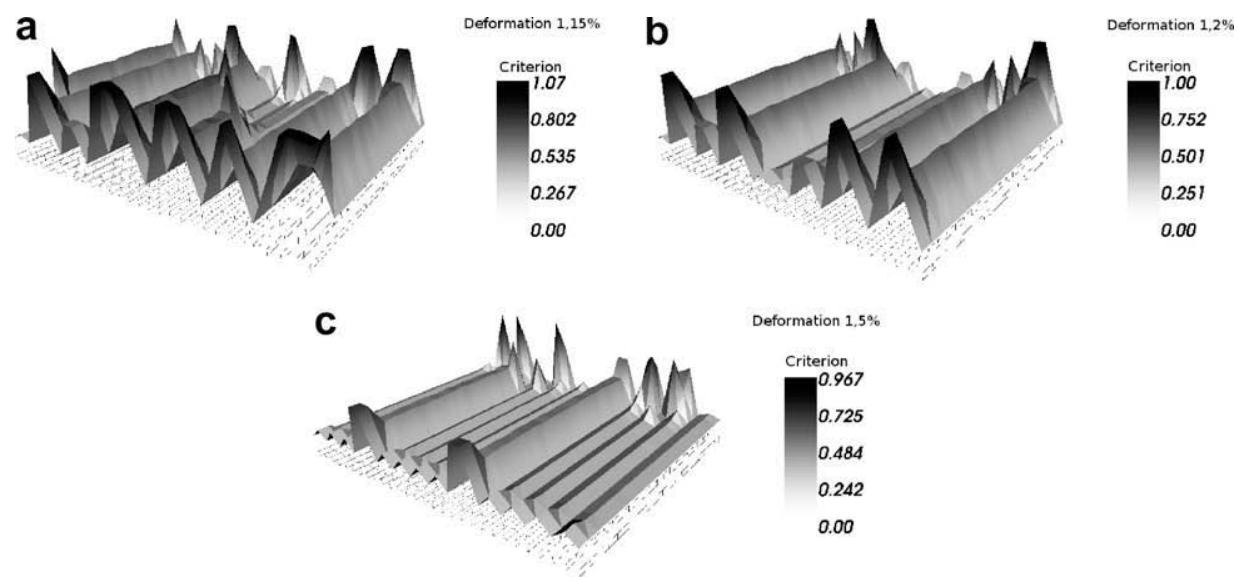

Fig. 9. Criterion in the $90^{\circ}$ ply for three loads in a $[0 / 90 / 0]$.

tial rupture surfaces is determined. Next, using the microscopic model, one seeks the surface which verifies the given criterion: this surface becomes ruptured and its behavior switches from that of a perfect interface to contact with friction. Then, the multiscale calculation is performed again. This process converges very rapidly (only a few iterations) because the degradation is localized. When the criterion is no longer verified by any surface, one increases the loading. This strategy is very robust because the loading step is linked directly to the size of a potential surface.

\section{Some results}

Our strategy required the development of a dedicated program in $\mathrm{C}++$, which enabled us to carry out some demonstrative simulations. We are going to focuss now on the numerical reproduction of classical experimental observations. Three elementary frameworks are illustrated: transverse loading on cross-ply laminates, shear loading on cross-ply laminates, and finally a notched sample.

\subsection{Example 1: transverse loading on cross-ply laminates}

This strategy along with the use of the micromodel enabled us to simulate classical experiments on $\left[0_{m}, 90_{n}\right]_{s}$ samples in which the thickness of the $90^{\circ}$ ply varies (Fig. 8). These samples were subjected to longitudinal strain. First, one can observe that the cracks for thick $90^{\circ}$-ply specimens are really unstable, whereas for thin plies some cracks initiate near the edge and do not propagate. As can be shown with micromechanics models, the degradation pattern is nearly periodic.

The energy recovery rates for each surface enable one to analyze the degradation. On the Fig. 9, we show the repartition of the criterion (varying from 0 to 1 ) over the laminate.

A quantitative study enabled us to come close to the experimental results by varying the thickness of the $90^{\circ}$ ply (Fig. 10).

Similarly, no additional assumption is necessary in order to simulate a $\left[90_{2} / 0\right]_{s}$ laminated composite. The cracks occur randomly in the two transverse plies. On Fig. 11, we can see an increasing number of transverse cracks in the $90^{\circ}$ plies due to the loading.

The key-point in these results is that they capture (at least from the qualitative point of view) the main observations of classical micromechanical experiments. On such simple geometries, these results could of course be obtained by well-known micromechanical semi-analytical models. However, these models would assume specific representation for each configuration (for example for middle of skin plies). It is here achieved in a completely automatic way, thus the method can be applied to much more complex structures.

\subsection{Example 2: shear loading on cross-ply laminates}

The second example deals with tensile testing of $\left[45_{n} /-45_{n}\right]_{m s}$ laminates. Such a test is well-known for inducing an almost pure shear state within the elementary ply. In this case, the global stress-strain curve is highly non-linear as illustrated Fig. 12. An-

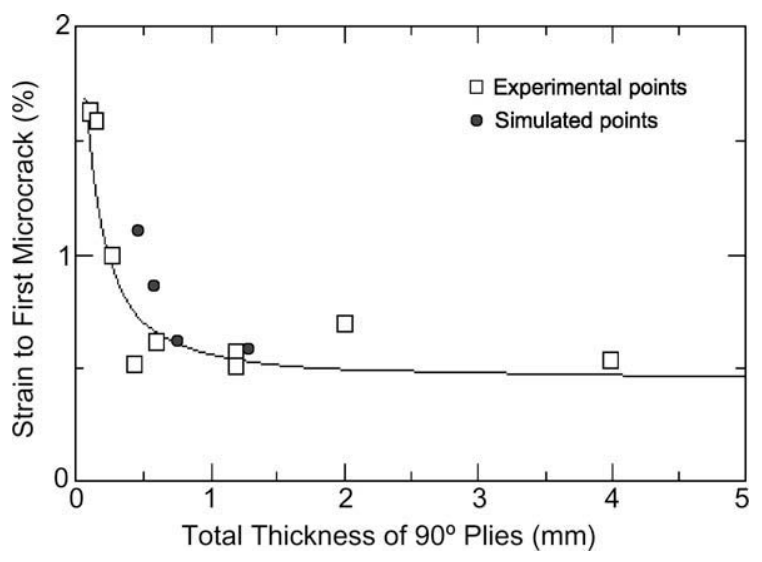

Fig. 10. Comparison of numerical and experimental results with respect to the influence of the thickness of the transverse ply. 

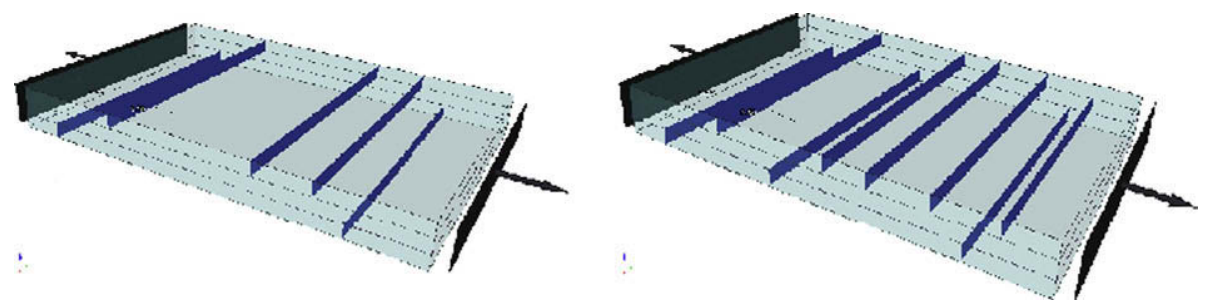

Fig. 11. Failure in the case of a $\left[90_{2} / 0\right]_{s} /$ laminate.
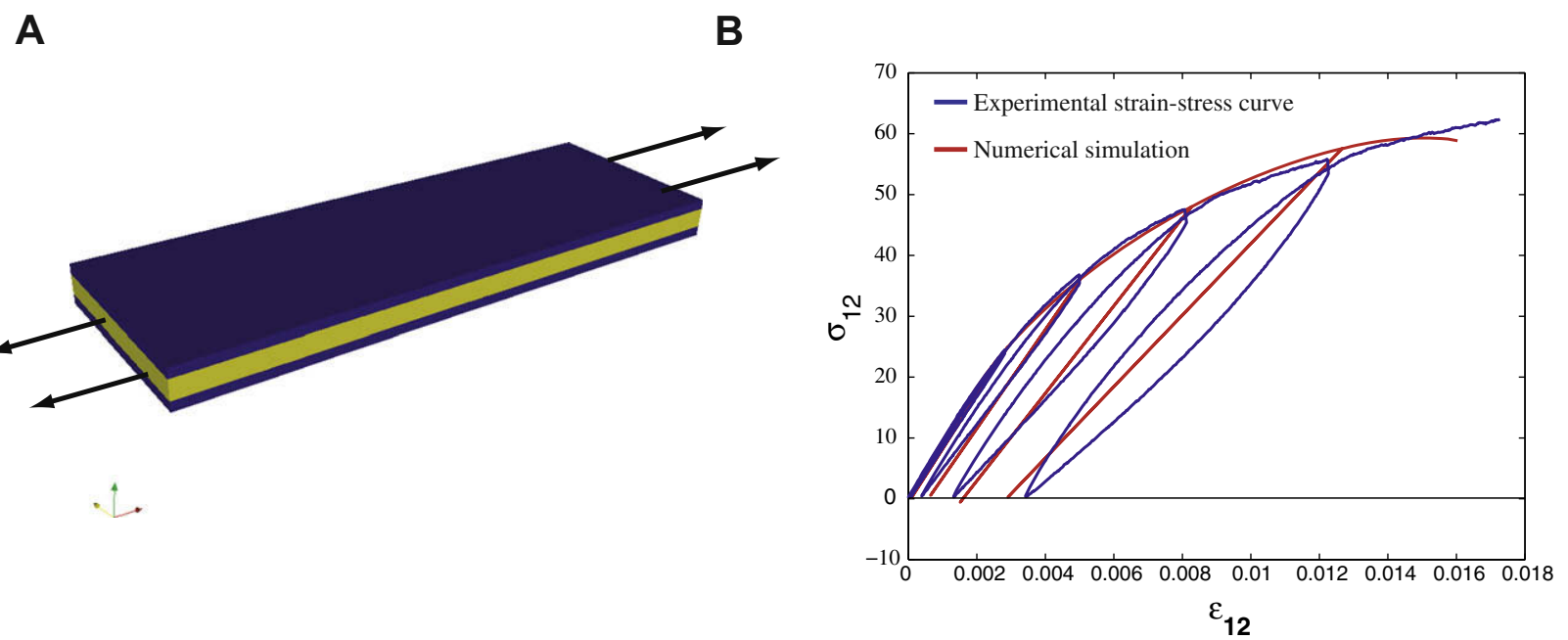

Fig. 12. (A) Tensile test on a $\left[45_{n} /-45_{n}\right]_{m s}$ laminate. (B) Global mechanical response with loading/unloading loops.
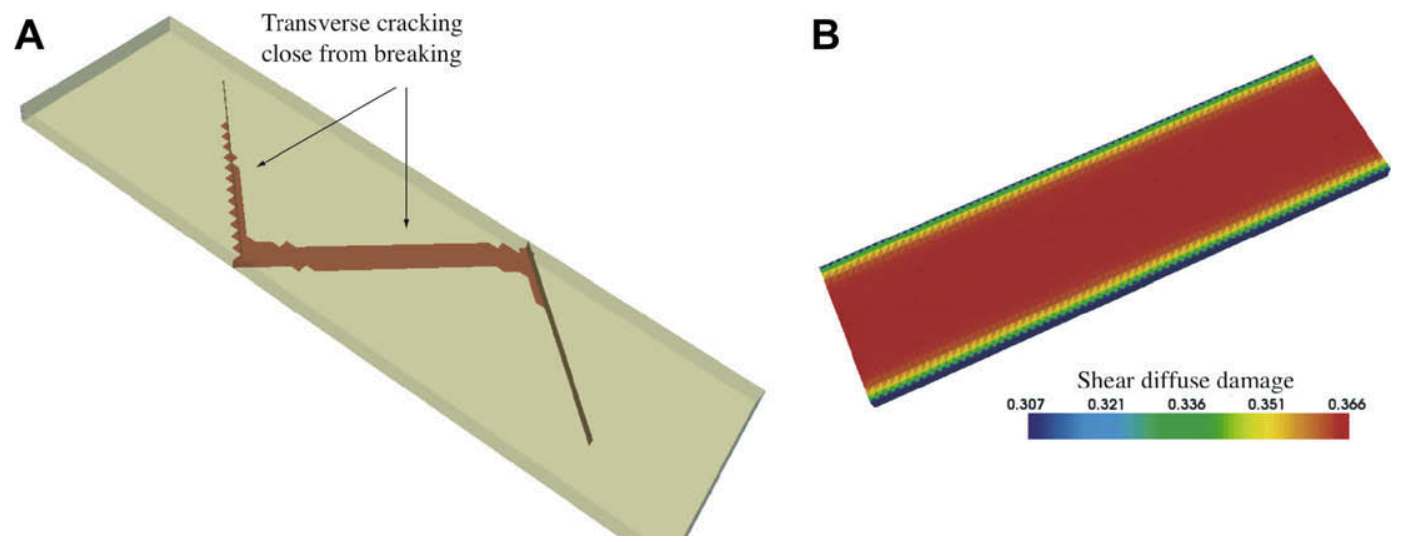

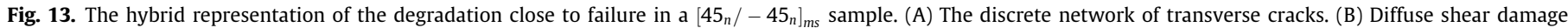
within the sample.

other key-point is that experimental observations clearly proved that the decreasing of apparent modulus can not be associated with discrete mechanisms, as transverse cracking. Indeed, discrete mechanisms will appear just prior breaking, their localization being responsible of the final breaking. But apparent level of damage up to 40 per cent can be observed before any localization.

The interest of the hybrid approach is clearly highlighted here. The shear behavior is related to the progressive debonding between carbon fibers and the epoxy matrix. It is well predicted as illustrated by the diffuse shear damage indicator of Fig. 13(b). On the other hand, Fig. 13(a) illustrates the pattern of transverse cracking just prior breaking, which is responsible for the final localization.

\subsection{Example 3: dual-notch sample}

The last result shows the full capabilities of the micro model. We simulated a dual-notch crossed sample subjected to longitudinal strain. The results are compared (see Fig. 14) to those of Beaumont [27]. Splitting at the crack tips can be observed very rapidly. As this splitting progresses, delamination occurs along this longitudinal crack at an angle $\alpha$ with the crack. Then, transverse cracking occurs at the level of the longitudinal crack. the results obtained are completely satisfactory, especially from a phenomenological point of view. The magnitudes of the applied forces, in the order of $200 \mathrm{~N}$, correspond relatively well to those determined experimentally. 


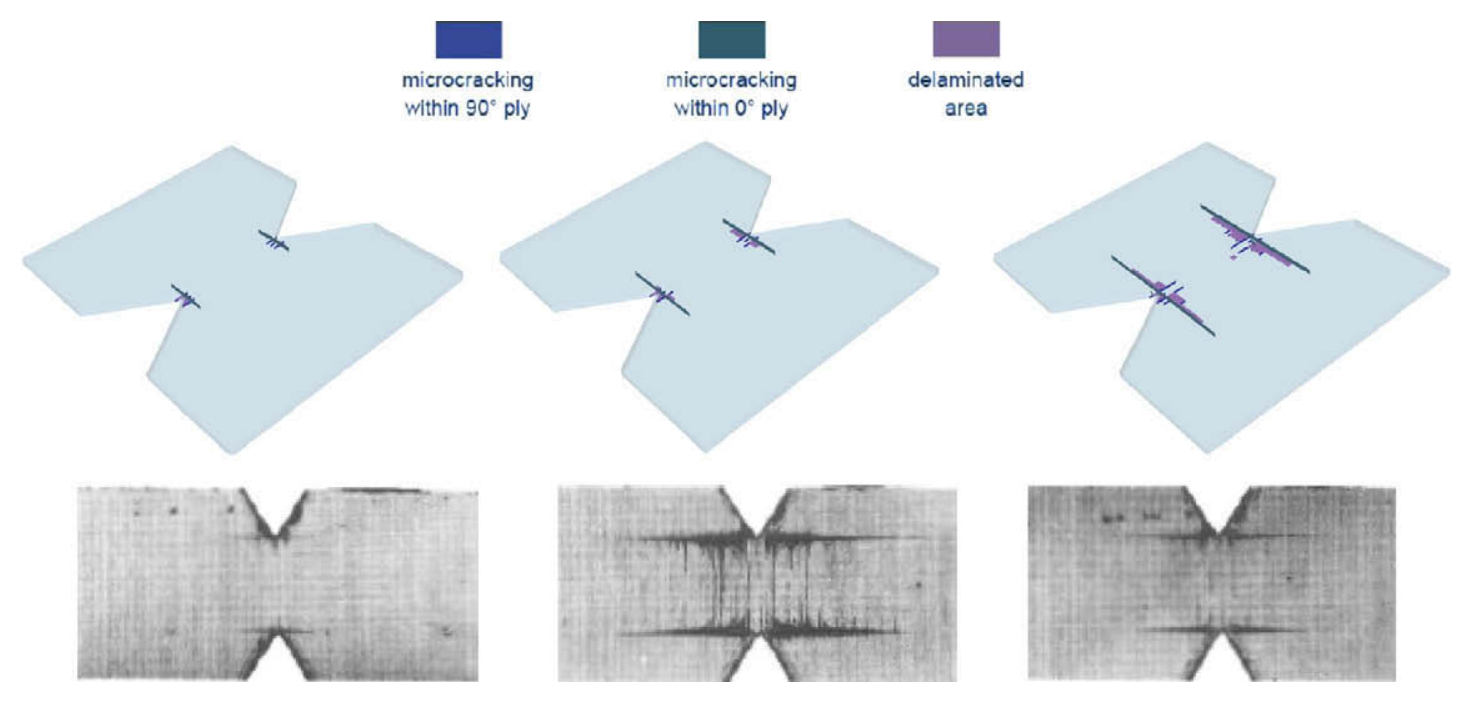

Fig. 14. A notched $[0,90]_{s}$ laminate.

\section{Conclusion}

A complete microscopic model was proposed in [1]. This model requires a special-purpose calculation strategy which we developed in this work. Our strategy, besides enabling one to carry out very large nonlinear calculations, also leads to a reduction in the calculation cost for the estimation of the criteria associated with potential rupture surfaces. The first results are very promising and lead to degradation patterns which other models are incapable of producing. The method must still be improved in order to accelerate the resolution and make simulations over longer periods possible. Damage within substructures remains to be implemented in order to be able to observe the coupling between diffuse damage and transverse cracking, particularly under shear loading. In the long run, this strategy should make the simulation of complex samples containing different orientations possible. Extensions of the microscopic model to take into account the environment and the loading (oxidation, fatigue, etc.) have already been proposed in [3] and their qualitative simulations appear promising.

This computational micromodel is seen as a virtual reference material, i.e. a data base which contains all information coming from the macro and the micro scales.

\section{Acknowledgement}

This work was carried out under the AMERICO project (Multiscale Analyses: Innovating Research for CFRP), directed by ONERA (the French Aeronautics and Space Research Center) and funded by the DGA/STTC (the French Ministry of Defense), whose support is gratefully acknowledged.

\section{References}

[1] Ladevèze P. Multiscale computational damage modelling of laminate composites. In: Sadowski T, editor. Multiscale modelling of damage and fracture processes in composite materials, CISM courses. Springer-Verlag; 2005. p. 171-212.

[2] Ladevèze P, Lubineau G, Violeau D. A computational damage micromodel of laminated composites. Int J Fract 2006;137(1-4):139-50.

[3] Lubineau G, Ladevèze P, Violeau D. Durability of cfrp laminates under thermomechanical loading: a micro-meso damage model. Compos Sci Technol 2006;66(7-8):983-92.

[4] Ladevèze P, LeDantec E. Damage modeling of the elementary ply for laminated composites. Compos Sci Technol 1992;43(3):257-67.

[5] Allix O, Ladevèze P. Interlaminar interface modelling for the prediction of delamination. Compos Struct 1992;22(4):235-42.
[6] Ladevèze P. On the damage mechanics of composites. In: Bathias C, Menkès D, editors. Comptes-rendus des JNC5. Paris: Pluralis Publication; 1986. p. 667-83. In French..

[7] Zhang H, Ericson M, Varna J, Berglund L. Transverse single-fibre test for interfacial debonding in composites: 1. Experimental observations. Compos A Appl Sci Manuf 1997;28A(4):309-15.

[8] Sjögren B, Berglund L. The effects of matrix and interface on damage in grp cross-ply laminates. Compos Sci Technol 2000;60(1):9-21.

[9] Lagattu F, Lafarie-Frénot M. Variation of PEEK matrix crystallinity in APC-2 composite subjected to large shearing deformations. Compos Sci Technol 2000;60(4):605-12.

[10] Boniface L, Smith P, Bader M, Rezaifard A. Transverse ply cracking in cross-ply cfrp laminates - initiation or propagation controlled ? J Compos Mater 1997;34(11):1080-112.

[11] Crossman F, Wang A. The dependence of transverse cracking and delamination on ply thickness in graphite/epoxy laminates. In: Reifsnider K, editor, Damage in composite materials. ASTM-STP 775, 1982; pages 118-139.

[12] Boniface L, Smith P, Ogin S, Bader M. Observations on transverse ply crack growth in a $\left[0 / 90_{2}\right]_{\mathrm{s}}$ CFRP laminate under monotonic and cyclic loading. Proc 6th Intl Conf Compos Mater 1987;3:156-65.

[13] Nairn J, Hu S. The initiation and growth of delaminations induced by matrix microcracks in laminated composites. Int J Fract 1992;57(1):1-24.

[14] Ogihara S, Takeda N. Interaction between transverse cracks and delamination during damage process in CFRP cross-ply laminates. Compos Sci Technol 1995;54(4):395-404.

[15] Zhang J, Fan J, Herrmann K. Delaminations induced by constrained transverse cracking in symmetric composite laminates. Int J Solids Struct 1999;36(6):813-46.

[16] Nairn J. Matrix microcracking in composites. In: Manson Taljera, editor. Polymer matrix composition. Comprehensive composite materials. Elsevier Science; 2000. p. 403-32. Ch.13.

[17] Berthelot J. Transverse cracking and delamination in cross-ply glass-fiber and carbon-fiber reinforced plastic laminates: static and fatigue loading. Appl Mech Rev 2003;56(1):111-47.

[18] Hashin Z. Analysis of cracked laminates: a variational approach. Mech Mater 1985;4:121-36.

[19] Ho S, Suo Z. Tunneling cracks in constrained layers. J Appl Mech 1993;60:890-4.

[20] Dvorak G, Laws N. Analysis of progressive matrix cracking in composite laminates part 2. First ply failure. J Compos Mater 1987;21:309-29.

[21] Ladevèze P, Loiseau O, Dureisseix D. A micro-macro and parallel computational strategy for highly heterogeneous structures. Int J Numer Methods Eng 2001;52:121-38.

[22] Ladevèze $P$, Nouy A, Loiseau $O$. A multiscale computational approach for contact problems. Comput Methods Appl Mech Eng 2002;191:4869-91.

[23] Ladevèze $P$, Nouy $A$. On a multiscale computational strategy with time and space homogenization for structural mechanics. Comput Methods Appl Mech Eng 2003;192:3061-88.

[24] Farhat C, Roux FX. A method of finite element tearing and interconnecting and its parallel solution algorithm. Int J Numer Methods Eng 1991;32:1205-27.

[25] Mandel J. Balancing domain decomposition. Commun Numer Methods Eng 1993;9:233-41.

[26] Nairn J. Exact and variational theorems for fracture mechanics of composites with residual stresses, traction-loaded cracks, and imperfect interfaces. Int J Fract 2000;105:243-71.

[27] Beaumont P. Damage accumulation. In: Talreja R, editor. Damage mechanics of composite materials. Composite materials series, Vol. 9. Amsterdam: Elsevier Science; 1994. p. 139-85. 